\section{Relação entre hábitos bucais, alterações oronasofaringianas e mal-oclusões em pré-escolares de Vitória, Espírito Santo, Brasil}

\author{
The relationship between oral habits, \\ oronasopharyngeal alterations, and malocclusion \\ in preschool children in Vitória, Espírito Santo, Brazil
}

Adauto Emmerich 1

Luiza Fonseca 2

Ana Maria Elias 3

Urubatan Vieira de Medeiros 4

\author{
${ }^{1}$ Núcleo de Pesquisas \\ Craniofaciais, Departamento \\ de Medicina Social, Centro \\ Biomédico, Universidade \\ Federal do Espírito Santo, \\ Vitória, Brasil. \\ 2 Fundação para \\ o Desenvolvimento da \\ Ciência-Odontologia, \\ Salvador, Brasil. \\ 3 Faculdade de Ciências \\ e Letras, Universidade \\ Estadual Paulista, \\ Araraquara, Brasil. \\ ${ }^{4}$ Departamento de \\ Odontologia Social \\ e Preventiva, Faculdade de \\ Odontologia, Universidade \\ Federal do Rio de Janeiro, \\ Rio de Janeiro, Brasil. \\ Correspondência \\ Adauto Emmerich \\ Av. Marechal Campos 1468, \\ Vitória, ES 29040-090, Brasil. \\ adautoemmerich@terra.com.br
}

\begin{abstract}
The objective of this study was to estimate the prevalence of malocclusion and associated variables such as deleterious habits (DH) and oronasopharyngeal alterations (OA), mouth breathing, atypical phonation, and atypical swallowing in three-year-old children in Vitória, Espírito Santo State, Brazil. The sample included 291 children of both sexes enrolled in a Children's Educational Center and selected through probability sampling by conglomerates. Logistic regression indicated a high relative risk $(R R)$ in children with altered overjet, open bite, and crossbite to present mouth breathing $(R R=1.89$; $C I$ : 1.56-2.03), $(R R=2.46$; $C I: 2.00-3.02),(R R=1.45$; $C I$ : 1.23-1.72); atypical swallowing $(R R=2.57$; $C I: 1.87-3.52),(R R=3.49 ; C I: 2.53-4.81),(R R=$ 1.86; CI: 1.46-2.39); and atypical phonation $(R R=$ 2.25; CI: 1.66-3.05), ( $R R=3.18$; $C I: 2.38-4.25)$, $(R R=1.71 ; C I: 1.32-2.22)$, respectively. An association was shown between finger or pacifier sucking and altered overjet $(p<0.001)$, and between pacifier sucking and open bite $(p<0.001)$. Such results indicate that the prevalence of malocclusions is associated with $\mathrm{DH}$ and $\mathrm{OA}$.
\end{abstract}

Malocclusion; Primary Dentition; Prevalence

\section{Introdução}

Para Moorrees et al. 1, a face do ser humano juntamente com a sua dentição funcionam harmonicamente como um espelho da expressão e da emoção, e têm fundamental importância na fala e na capacidade de comunicação. Assim, o tratamento das mal-oclusões e das desarmonias oclusais deveria ser considerado dentro da área de atenção dos serviços de saúde pública, em decorrência das implicações fisiológicas integradas da boca. Além disso, Shaw et al. 2 admitiram ser as mal-oclusões responsáveis por algum transtorno na personalidade, causados por apelido, gozação, ridicularização e insulto, que podem predispor a uma baixa auto-estima e à alienação social.

Hanson \& Cohen ${ }^{3}$ consideraram as modificações iniciais na fonação, deglutição, respiração bucal (RB), sucção de dedo e de chupeta como sinais clínicos iniciais de futuras mal-oclusões. Behlfelt 4 associou a mudança de postura da cabeça, osso hióide, língua, dimensão da faringe e a RB à mordida cruzada. Vanetikidou 5 relacionou também a freqüência de mordida cruzada com a RB, ocorrendo uma influência negativa de fatores ambientais sobre a região craniofacial.

Fränkel \& Fränkel 6 consideraram também que um adequado selamento bucal anterior, associado a um balanço postural no sinergismo dos vários grupos musculares dos lábios da 
face, deve ser visto como um indicador do estabelecimento da maturação do comportamento postural de todo o complexo orofacial. Moyers \& Carlson 7 assinalaram que fatores, como crescimento dos músculos, sua migração e inserções, as variações da função neuromuscular e a função anormal da RB, influenciam marcadamente alguns aspectos do crescimento e da formação craniofacial.

O presente estudo tem por objetivos avaliar, nos pré-escolares matriculados nos Centros de Educação Infantil Municipais de Vitória, Espírito Santo, a prevalência de mal-oclusões e associar as variáveis oclusais (VO), mordida cruzada, mordida aberta e sobressaliência alterada com hábitos deletérios (HD) e alterações oronasofagianas (AO) (RB, e problemas relacionados com a deglutição e a fala).

\section{Material e métodos}

\section{População alvo do estudo}

Este estudo foi realizado no ano de 2000, na cidade de Vitória, capital do Estado do Espírito Santo, Sudeste do Brasil. A população alvo do estudo consistiu em todas as crianças de três anos de idade que freqüentavam os Centros de Educação Infantil (CEIs) do município. A pesquisa foi dividida em duas etapas: na primeira, estabeleceu a prevalência das mal-oclusões, das variáveis oclusais, sobressaliência, sobremordida e mordida cruzada, e das AO (RB, deglutição e fonação atípica); e na segunda, com entrevista familial, procurando evidenciar os hábitos e costumes individuais relacionados com o desenvolvimento de possíveis problemas oclusais.

\section{Desenho da amostra}

A amostra foi selecionada por processo de amostragem por conglomerado. Para cálculo do tamanho da amostra foram tomados como referência os valores de prevalência de maloclusões observados em pesquisa realizada pela Universidade de São Paulo, Faculdade de Saúde Pública/Secretaria de Estado da Saúde de São Paulo 8, no Município de São Paulo, para a idade de cinco anos $(49,2 \%)$ e a prevalência aos três anos $(80,2 \%)$ obtida em pesquisa realizada por Martins et al. 9, no Município de Araraquara, São Paulo. Para efeito do cálculo, considerou-se a prevalência nessa idade igual a $70,0 \%$. Com esse valor, admitindo-se um erro máximo de sete pontos percentuais e confiança de $95,0 \%$ na estimativa da prevalência, con- tando-se com uma perda potencial de $20,0 \%$ dos elementos da amostra e com efeito do desenho (deff) igual a 1,4, o tamanho final da amostra ficou estabelecido em 289 crianças. Foram selecionadas 17 unidades escolares (CEIs) por meio de amostragem sistemática com probabilidade proporcional ao tamanho e, dentro de cada unidade foram selecionadas 17 crianças por meio de amostragem casual simples.

\section{Calibração dos examinadores}

O exame clínico foi realizado por cinco examinadores cirurgiões-dentistas, previamente treinados e calibrados. Para avaliar a efetividade da calibração, 12 crianças previamente selecionadas em uma creche não pertencente à amostra, foram avaliadas pelos cinco examinadores com relação aos 23 itens relativos ao exame clínico. O grau de concordância nas classificações foi medido por meio da estatística kappa. Os resultados mostraram, tanto na primeira réplica quanto na segunda, um alto grau de concordância nas classificações em cada item considerado (kappa $>0,5 ; \mathrm{p}<0,001$ ).

\section{Variáveis do estudo}

Além das medidas relativas à oclusão dental, foram registrados o sexo e a origem racial expressada pela cor da criança. Crianças de cor amarela não foram analisadas, pois conta com somente $0,6 \%$ na população estudada. Os critérios para se estabelecer a mal-oclusão foram adotados de acordo com Angle 10 e Baume 11, modificados por Maia 12, da seguinte forma: (1) caninos e molares em relações normais, ou seja, canino superior ocluindo entre o canino inferior e o primeiro molar decíduo; (2) ausência de mordidas cruzadas anterior ou posterior; (3) sobremordida de $\pm 1 \mathrm{~mm}$ sem exceder um terço da coroa do incisivo inferior; (4) sobressaliência de $\pm 1 \mathrm{~mm}$ e não exceder $3 \mathrm{~mm}$; (5) ausência de apinhamentos; (6) todos os dentes decíduos presentes na cavidade bucal; (7) relação terminal dos segundos molares decíduos em plano vertical ou em degrau mesial para a mandíbula; (8) ausência de anomalias dentárias de forma e número.

Para a sobremordida pediu-se aos pacientes que colocassem os dentes em oclusão cêntrica e observou-se o sobrepasse dos dentes anteriores superiores sobre os inferiores. Registrou-se: como normal, com variação de 2 a $3 \mathrm{~mm}$; profunda, com o incisivo inferior tocando o palato; moderada, com uma variação acima de $3 \mathrm{~mm}$, sem tocar o palato; mordida topo a topo, com os bordos incisais dos incisivos su- 
periores tocando nos bordos incisais dos incisivos inferiores. A mordida aberta anterior foi registrada, caso existisse uma falta de sobreposição vertical entre quaisquer dos incisivos antagonistas, sem estimar a quantidade de mordida aberta. Na sobressaliência com os dentes em oclusão cêntrica, observou-se a distância horizontal entre as faces lingual dos incisivos da maxila e a vestibular dos incisivos da mandíbula. O valor normal foi atribuído a uma distância até $3 \mathrm{~mm}$; aumentada, de $3,1 \mathrm{~mm}$ a $5 \mathrm{~mm}$; exagerada, acima de $5,1 \mathrm{~mm}$ e negativa, para os indivíduos com protusão da mandíbula (classe III de Angle 10). A sobressaliência não foi registrada nos casos em que todos os incisivos superiores e/ou inferiores estivessem ausentes. Na mordida cruzada, com os dentes em oclusão cêntrica, a mordida cruzada anterior foi registrada quando um ou mais de um dos incisivos superiores apresentassem uma inclinação axial anormal, ocluindo lingualmente, em relação aos seus antagonistas, os incisivos inferiores. A mordida cruzada posterior foi registrada, considerando quando dois ou mais dentes posteriores, incluindo-se também os caninos, apresentassem um problema oclusal em que as cúspides vestibulares dos dentes superiores ocluíam lingualmente em relação às cúspides vestibulares dos dentes inferiores. Foi considerada a mordida cruzada posterior uni ou bilateral, entretanto a mordida cruzada funcional, observada com um deslocamento da mandíbula, não foi registrada em decorrência da dificuldade do diagnóstico em termos de estudo epidemiológico.

Para o exame das AO foi solicitado: (1) para a RB, que o paciente fizesse flexões de joelhos com os lábios fechados, fazendo entre 10 e 15 flexões sem respirar pela boca, o que foi considerado como um bom espaço aéreo nasal 6 . Os examinadores solicitaram também, com espelhos posicionados na entrada do espaço aeronasal, que a criança expirasse o ar com bastante força. A criança que não conseguiu marcar com ar o espelho e apresentou algum tipo de dificuldade respiratória e/ou obstrução nasal e sem vedamento labial foi considerada RB; (2) o teste da deglutição foi realizado com a criança sentada em uma cadeira e o examinador solicitou-lhe que ingerisse um pouco d'água e observou, na deglutição atípica, os seguintes movimentos: mímica com os músculos dos lábios, pressão das comissuras labiais, interposição da língua entre os dentes e ausência de contração dos masséteres 13; e (3) na avaliação da fonação, foi solicitado às crianças que repetissem palavras com os fonemas /s/ e /z/, observando a pronúncia. A fonação foi atípica, quando hou- ve projeção da língua anteriormente ou lateralmente no momento da articulação dos fonemas 14 .

\section{Análise estatística}

A estimação da prevalência de mal-oclusão foi feita por intervalos de confiança (IC) de 95\%. Para a verificação da existência de associação entre VO e HD, foram utilizadas as medidas usualmente empregadas, como o teste do quiquadrado para tabelas de contingência e a estimação do RR por meio da regressão logística.

Este estudo foi aprovado pelo Comitê de Ética em Pesquisa do Centro Biomédico da Universidade Federal do Espírito Santo, conforme parecer do Extrato da 21a Ata da Reunião Ordinária da Comissão de Ética, em 04 de agosto de 1999.

\section{Resultados}

Na primeira fase da pesquisa, em que foram realizados exames clínicos, visando a estimar as alterações oclusais, o número de indivíduos selecionados foi igual ao estabelecido no planejamento da amostra (291) crianças. Na segunda fase, em que foram realizadas entrevistas com as mães das crianças examinadas, para estabelecer alguns aspectos etiológicos, a perda esteve dentro dos limites previstos (231 crianças).

Das 291 crianças de três anos examinadas na primeira fase da pesquisa, $59,1 \%$ apresentaram algum tipo de mal-oclusão. Isso permite dizer que a prevalência de mal-oclusões entre crianças de três anos, freqüentando CEIs, em Vitória, Espírito Santo, encontra-se entre 53,3\% e $64,9 \%$. A prevalência de sobressaliência alterada encontra-se em torno de $40,0 \%$. A mordida aberta atingiu $25,8 \%$ e a mordida cruzada está presente em 12,0\% das crianças. Na Tabela 1 são apresentadas as prevalências estimadas das mal-oclusões consideradas no estudo, por ponto e por intervalo de confiança.

Na Tabela 2 são apresentados dados relativos à associação da mal-oclusão, em geral, com sexo e cor da pele, e das variáveis sobressaliência, sobremordida, mordida cruzada com sexo. Como pode ser notado, há evidência estatística para se admitir que as mal-oclusões observadas aos três anos não estão relacionadas ao sexo. Quanto à origem racial expressada na cor, o pequeno número de negros presentes na amostra $(11,0 \%)$, cor branca com $37,5 \%$ e a parda com $51,5 \%$, permitiu apenas que fosse comparada a cor com mal-oclusões por meio da clas- 


\section{Tabela 1}

Estimativas da prevalência de mal-oclusão em crianças de três anos, por ponto e por meio de intervalos de confiança de 95\%, para cada tipo de mal-oclusão. Vitória, Espírito Santo, Brasil, 2001.

\begin{tabular}{|c|c|c|}
\hline Tipo de mal-oclusão & Mal-oclusão (\%) & IC95\% \\
\hline Mal-oclusão* & $59,1^{\star \star}$ & $53,3-64,9$ \\
\hline \multicolumn{3}{|l|}{ Sobressaliência } \\
\hline Normal & 60,1 & $54,7-65,5$ \\
\hline Aumentada & 12,4 & $8,9-15,8$ \\
\hline Topo a topo & 2,1 & $0,4-3,7$ \\
\hline Exagerada & 8,9 & $5,5-12,3$ \\
\hline Negativa & 2,4 & $0,6-4,2$ \\
\hline \multicolumn{3}{|l|}{ Sobremordida } \\
\hline Normal & 49,5 & $42,0-56,9$ \\
\hline Moderada & 11,7 & $5,8-17,6$ \\
\hline Profunda & 7,9 & $4,0-11,8$ \\
\hline Topo a topo & 3,1 & $1,1-5,1$ \\
\hline Mordida aberta & 25,8 & $20,8-30,8$ \\
\hline \multicolumn{3}{|l|}{ Mordida cruzada } \\
\hline Ausente & 88,0 & $83,7-92,3$ \\
\hline Anterior & 3,4 & $1,2-5,6$ \\
\hline Posterior unilateral & 5,8 & $2,7-8,9$ \\
\hline Anterior e posterior & 1,4 & $0,2-2,6$ \\
\hline Posterior bilateral & 1,4 & $-2,9$ \\
\hline
\end{tabular}

* Somatório de todos os tipos de mal-oclusões encontradas.

** Em negrito os valores utilizados na análise estatística da Tabela 5. sificação preconizada pela Organização Mundial da Saúde (OMS) em 1987 15; mal-oclusão normal, leve e moderada ou severa. Neste caso, há evidência estatística de associação entre cor e mal-oclusão.

Os resultados do estudo da associação de maloclusões com HD, mostram haver associação estatisticamente significante de sucção de chupeta com sobressaliência $(\mathrm{gl}=1 ; \mathrm{p}<0,001)$ e sobremordida $(\mathrm{gl}=2 ; \mathrm{p}<0,001$ ) (Tabela 3$)$. Pode-se notar que é maior a proporção de crianças com sobressaliência alterada entre aquelas que usam ou usaram chupeta $(44,0 \%)$ que entre aquelas que nunca usaram (18,9\%). O mesmo é observado em relação à mordida aberta (40,5\% e $8,7 \%$, respectivamente).

Há associação estatística também entre sucção de dedo e sobressaliência ( $\mathrm{gl}=1, \mathrm{p}<0,002$ ), indicando que a proporção de crianças com sobressaliência alterada é maior entre aquelas com hábito de sucção de dedo que entre aquelas que não apresentam esse hábito (Tabela 4).

Pode-se observar, por meio do risco relativo (RR), que a chance de crianças com três anos de idade com sobressaliência alterada apresentarem $\mathrm{AO}$, como a RB (RR = 1,89; IC: $1,56-2,03)$, deglutição atípica $(\mathrm{RR}=2,57$; IC: $1,87-3,52) \mathrm{e}$ fonação atípica (RR = 2,25; IC: 1,66-3,05) é, pelos menos, 50,0\% maior que a daquelas com sobressaliência normal. Da mesma forma, o risco de uma criança com mordida aberta apresentar as AO é sempre maior que o dobro da chance de uma criança com sobremordida normal. Para a mordida cruzada, a chance é pelo menos $23,0 \%$ maior que a de uma criança com ausência de mordida cruzada (Tabela 5).

\section{Discussão}

As mal-oclusões têm sido evidenciadas como uma das doenças da civilização moderna, como são a diabete e as doenças coronarianas, com vários agentes concebíveis, como a alergia respiratória, dieta macia, perda prematura de dentes decíduos, ausência de amamentação, RB e HD 16,17,18.

O presente estudo demonstrou ser alta a prevalência das mal-oclusões para a idade de três anos $(59,1 \%)$. Embora uma análise comparativa com outros estudos seja difícil, em decorrência de desenhos de estudo bastante discrepantes e critérios diferenciados de classificação adotados, observa-se um aumento das mal-oclusões na população mundial para a idade de três anos, não havendo evidência estatística de ocorrência diferenciada de mal-oclusão entre os sexos e a cor 9,12,19,20,21,22. Em relação 
Classificação das crianças de três anos segundo sobressaliência e sobremordida, em relação ao hábito de sucção de chupeta. Vitória, Espírito Santo, Brasil, 2001.

\begin{tabular}{|c|c|c|c|c|c|c|c|}
\hline \multirow[t]{3}{*}{ Variável } & \multicolumn{4}{|c|}{ Sucção de chupeta } & \multicolumn{2}{|c|}{ Total } & \multirow[t]{3}{*}{$p$} \\
\hline & \multicolumn{2}{|c|}{ Não } & \multicolumn{2}{|c|}{ Sim } & & & \\
\hline & $n$ & $\%$ & $n$ & $\%$ & $\mathrm{n}$ & $\%$ & \\
\hline Sobressaliência & & & & & & & $<0,001$ \\
\hline Normal & 90 & 81,1 & 47 & 56,0 & 137 & 70,3 & \\
\hline Alterada & 21 & 18,9 & 37 & 44,0 & 58 & 29,7 & \\
\hline Total & 111 & 56,9 & 84 & 43,1 & 195 & & \\
\hline Sobremordida & & & & & & & $<0,001$ \\
\hline Normal & 78 & 67,8 & 39 & 35,1 & 117 & 51,8 & \\
\hline Aberta & 10 & 8,7 & 45 & 40,5 & 55 & 24,3 & \\
\hline Outros & 27 & 23,5 & 27 & 24,3 & 54 & 23,9 & \\
\hline Total & 115 & 50,9 & 111 & 49,1 & 226 & & \\
\hline
\end{tabular}

Tabela 4

Classificação das crianças de três anos segundo sobressaliência e hábito de sucção de dedo. Vitória, Espírito Santo, Brasil, 2001.

\begin{tabular}{|c|c|c|c|c|c|c|c|}
\hline \multirow[t]{3}{*}{ Variável } & \multicolumn{4}{|c|}{ Sucção de dedo } & \multicolumn{2}{|c|}{ Total } & \multirow[t]{3}{*}{$p$} \\
\hline & \multicolumn{2}{|c|}{ Não } & \multicolumn{2}{|c|}{ Sim } & & & \\
\hline & $\mathrm{n}$ & $\%$ & $n$ & $\%$ & $\mathrm{n}$ & $\%$ & \\
\hline Sobressaliência & & & & & & & $<0,002$ \\
\hline Normal & 128 & 73.1 & 9 & 45.0 & 137 & 70,3 & \\
\hline Alterada & 47 & 26.9 & 11 & 55.0 & 58 & 29,7 & \\
\hline Total & 175 & 89.7 & 20 & 10.3 & 195 & & \\
\hline
\end{tabular}

à raça, Freeman 23 considera que não existiu, e não existe, na espécie humana, uma população homogênea quanto à sua genética e à sua raça, e que o conceito biológico de raça é insustentável e não tem legitimidade nas ciências biológicas, sendo a raça um produto social e político das nações. Neste estudo obteve-se associação entre a classificação da OMS de 198715 e a cor pelo número pequeno de negros presentes na amostra $(11,0 \%)$.

A etiopatogenicidade das mal-oclusões é multifatorial, com uma interação de fatores congênitos, morfológicos, biomecânicos e ambientais com AO, como a RB, deglutição e fonação atípica 19,14.

Para Moyers 14, os aspectos funcionais associados à mastigação e à deglutição têm importante papel na arquitetura da boca e da região craniofacial. Graber 24 avaliou que a tría- de: duração, intensidade e freqüência do hábito de sucção de dedo, poderá influenciar o desenvolvimento craniofacial da criança, promovendo alterações neuromusculares, produzindo mordida cruzada, mordida aberta, sobressaliência alterada e deglutição atípica.

Muito embora não haja comprovação, é bem possível que o argumento para o crescente hábito de sucção de chupeta esteja na correspondente produção pelo sistema nervoso central de neurotransmissores (endorfinas) ligados à sensação de prazer 25,26 . A chupeta, um corpo estranho elástico, estando na boca, ativa a sucção, a salivação e a deglutição, saturando de informações aferentes o sistema funcional da alimentação. Se a causa do choro do bebê é a sede ou a fome, a chupeta consubstancia uma fraude, porque engana o organismo, produzindo saciedade sensorial, sem proporcio- 
Tabela 5

Estimativas de risco relativo (RR) de ocorrência de $\mathrm{AO}$ em crianças de três anos relacionado a mal-oclusões. Vitória, Espírito Santo, Brasil, 2001.

\begin{tabular}{lcc}
\hline Variável & RR & IC95\% \\
\hline Sobressaliência* & & \\
Respiração bucal & 1,89 & $1,56-2,03$ \\
Deglutição atípica & 2,57 & $1,87-3,52$ \\
Fonação atípica & 2,25 & $1,66-3,05$ \\
& & \\
Sobremordida* & 2,46 & $2,00-3,02$ \\
Respiração bucal & 3,49 & $2,53-4,81$ \\
Deglutição atípica & 3,18 & $2,38-4,25$ \\
Fonação atípica & & \\
Mordida cruzada* & 1,45 & $1,23-1,72$ \\
Respiração bucal & 1,86 & $1,46-2,39$ \\
Deglutição atípica & 1,71 & $1,32-2,22$ \\
Fonação atípica & 1,11 & \\
\hline
\end{tabular}

* Alterações oclusais dicotomizadas entre alterada e normal, aberta e normal, e presente e ausente, respectivamente. nar o respectivo aporte metabólico e competindo com o que seria ideal, a sucção e amamentação no seio materno. Além disso, tem ação prejudicial sobre o crescimento e desenvolvimento craniofacial, porque interfere nas relações posturais e dinâmicas de seus componentes, inibindo circuitos morfogenéticos 25 . Por outro lado, Bayardo et al. 27 consideraram que a ausência dos pais na integração da família é um fator significante na ocorrência de hábitos orais.

Os achados do presente estudo consideram a sucção de dedo e de chupeta estatisticamente significantes $(p<0,001)$, em relação às maloclusões, estando de acordo com Trombelli et al. 19, Øgaard et al. 28, Farsi et al. 29, Serra-Negra et al. 30, Tomita 21, Zuanon 31 . O significado etiológico do predomínio do uso da chupeta na atualidade pode ser atribuído ao modo de vida moderno, à crescente industrialização e a aspectos socioculturais 32,33 , haja vista que os estudos de Johnson 34 e Graber 24 enfatizaram, em sua época, o hábito de sucção de dedo, ou morder os lábios, não mencionando o uso da chupeta.

Para Moyers \& Carlson 7, a respiração nasal é um dos reflexos congênitos não condicionados ativos na região orofaríngea para que o recém-nascido sobreviva, assim como a sucção infantil e a deglutição. Um exemplo oposto é quando ocorre alguma obstrução nasal persistente que impede a respiração por essa via, sendo a criança condicionada a respirar pela boca, passando a realizar a $\mathrm{RB}$ como um reflexo condicionado (adquirido) indesejável.

Segundo Ricketts 35, Linder-Aronson 36, Cheng et al. 37 , Fränkel \& Fränkel 6 e Moyers \& Carlson 7 , o padrão respiratório alterado exerce influências sobre a morfologia facial e a dentição. Está relacionado com condições do clima, alergias, asmas, rinites, sinusites, pólipos nasais e atresias congênitas das coanas 5,38.

No presente estudo, observou-se que as $\mathrm{AO}$, RB (RR = 1,89; IC: 1,56-2,03), deglutição atípica $(R R=2,57$; IC: $1,87-3,52)$ e fonação atípica $(R R=$ 2,25; IC: 1,66-3,05) são fatores de risco em relação à sobressaliência alterada, sendo também fatores de risco para a mordida aberta, com a $\mathrm{RB}(\mathrm{RR}=2,46$; IC: 2,00-3,02), deglutição atípica $(R R=3,49 ;$ IC: $2,53-4,81)$ e fonação atípica $(R R=$ 3,18; IC: 2,38-4,25) e também fator de risco para a mordida cruzada presente, com a $\mathrm{RB}(\mathrm{RR}=$ 1,45; IC: 1,23-1,72), deglutição atípica ( RR = 1,86; IC: $1,46-2,39)$ e fonação atípica $(R R=1,71$; IC: 1,32-2,22) (Tabela 5). Águila-Ramos et al. 39 e Trombelli et al. 19 obtiveram resultados semelhantes aos deste estudo, muito embora não tenham feito análise de regressão logística. Entretanto, para Tomita 21 , a fonação atípica não apresenta relação com mal-oclusão. Hanson \& Cohen 3 , ao analisarem o efeito da forma e da função no desenvolvimento da dentição decídua, consideraram as modificações na fonação, deglutição, RB, sucção de dedo e de chupeta como sinais clínicos iniciais de futuras alterações oclusais. Tomita 21 não obteve diferenças estatisticamente significantes entre a mordida aberta anterior e a RB, estando, porém, a mordida aberta fortemente associada à sucção de chupeta. Alguns experimentos laboratoriais realizados por Harvold et al. 40 e Miller et al. 41 confirmaram a relação entre a RB e as maloclusões, com alterações na neuromusculatura orofacial. Miller et al. 41 recomendaram cautela para a extrapolação desses achados com as alterações que a RB provoca em humanos.

Concluindo, a prevalência das mal-oclusões na cidade de Vitória, Espírito Santo, apresentou, para a idade de três anos, valor epidemiológico alto $(59,1 \%)$ em comparação com a literatura estudada. Houve associação entre as mal-oclusões e HD e as AO. A chance de uma criança, na população estudada, com sobressaliência alterada, mordida aberta e mordida cruzada, apresentar alterações funcionais do sistema estomatognático é sempre maior do que em relação às crianças com oclusão normal. Com base nas evidências levantadas, devem-se incluir no planejamento e na organização dos programas e serviços públicos de saúde bucal, medidas profiláticas e de promoção 
de saúde voltadas para essa área, com uma perspectiva multiprofissional e interdisciplinar. Posteriormente, os tratamentos dos problemas oclusais mais simples, baseados em diagnóstico etiológico, morfológico e funcional, devem ser iniciados em idades precoces.

\section{Resumo}

O objetivo deste estudo foi estimar a prevalência das mal-oclusões e variáveis a elas associadas, como hábitos deletérios (HD) e as alterações oronasofaringianas (AO), respiração bucal, deglutição atípica e fonação atípica, em crianças com idade de três anos, no Município de Vitória, Espírito Santo, Brasil. A amostra constituiu-se de 291 crianças de ambos os sexos, matriculadas nos Centros de Educação Infantil, selecionadas por meio de amostragem probabilística por conglomerados. A análise de regressão logística indicou maior risco relativo (RR) de crianças com sobressaliência alterada, mordida aberta e mordida cruzada, em apresentar: respiração bucal ( $R R=1,89$; IC: 1,56-2,03), $(R R=$ 2,46; IC: 2,00-3,02), (RR = 1,45; IC: 1,23-1,72); deglutição atípica $(R R=2,57 ; I C: 1,87-3,52),(R R=3,49$; IC: 2,53-4,81), (RR = 1,86; IC: 1,46-2,39) e fonação atípica ( $R R=2,25 ; I C: 1,66-3,05),(R R=3,18 ; I C: 2,38-4,25)$, $(R R=1,71 ; I C: 1,32-2,22)$, respectivamente. Foi mostrado haver associação entre sucção de dedo e de chupeta com sobressaliência alterada $(p<0,001)$ e sucção de chupeta e mordida aberta $(p<0,001)$. Esses resultados indicam que a prevalência das mal-oclusões está associada aos HD e às AO.

Mal-oclusão; Dentição Decídua; Prevalência

\section{Colaboradores}

L. Fonseca contribuiu na definição de variáveis a serem trabalhadas e na leitura final do artigo, dando valiosas sugestões. A. M. Elias participou no planejamento e na análise estatística dos dados do artigo e dos resultados produzidos. U. V. Medeiros colaborou na definição de variáveis a serem trabalhadas e na leitura final do artigo, dando sugestões. A. Emmerich participou de todas as etapas da elaboração do artigo.

\section{Agradecimentos}

Os autores expressam seus sinceros agradecimentos ao Programa Institucional de Capacitação Docente e Técnica da Coordenação de Aperfeiçoamento de Pessoal de Nível Superior, pela concessão de Bolsa de Estudos para o desenvolvimento deste trabalho de Doutorado do Prof. Dr. Adauto Emmerich Oliveira, na Universidade Federal do Rio de Janeiro, em dezembro de 2001. Ao Secretário Municipal da Saúde de Vitória, Espírito Santo, Dr. Anselmo Tose, pela cessão das cirurgiãs-dentistas, Dra. Fátima Boni, Dra. Sônia Maria Beltrame, Dra. Sorlene Maria Martinelli e Dra. Vânia Azevedo de Souza, que contribuíram no desenvolvimento da pesquisa de campo e na coleta dos dados das amostras. 
1. Moorrees CFA, Burstone CJ, Cristiansen RL Hixon EH, Weinstein S. A "State-of-the-art" workshop conducted by the Oral-Facial Growth and Development Program, The National Institute of Dental Research. Am J Orthod 1971; 59:1-18.

2. Shaw WC, Meek SC, Jones DS. Nicknames, teasing, harassment and the salience of dental features among school children. Br J Orthod 1980; 7:75-80.

3. Hanson ML, Cohen MS. Effects of form and function on swallowing and the developing dentition. Am J Orthod 1973; 64:63-82.

4. Behlfelt K. Enlarged tonsils and the effect of tonsillectomy. Swed Dent J Suppl 1990; 72:1-35.

5. Venetikidou A. Incidence of malocclusion in asthmatic children. J Clinic Pediatr Dent 1993; 17:8994.

6. Fränkel R, Fränkel CH. Ortopedia orofacial. São Paulo: Editora Santos; 1990.

7. Moyers RE, Carlson DS. Maturação da neuromusculatura orofacial. In: Enlow DH, organizador. Crescimento facial. São Paulo: Artes Médicas; 1993. p. 260-3.

8. Núcleo de Estudos e Pesquisas de Sistemas de Saúde, Faculdade de Saúde Pública, Universidade de São Paulo/Secretaria de Estado da Saúde de São Paulo. Levantamento epidemiológico da cárie dentária, oclusopatias e fluorose dentária, em crianças de 5 a 12 anos de idade, em escolas públicas e privadas do Município de São Paulo, em 1996. São Paulo: Núcleo de Estudos e Pesquisas de Sistemas de Saúde, Faculdade de Saúde Pública, Universidade de São Paulo/Secretaria de Estado da Saúde de São Paulo; 1997. (Relatório técnico).

9. Martins JCR, Sinimbú CMB, Dinelli TCS, Martins LPM, Raveli DB. Prevalência de má-oclusão em pré-escolares de Araraquara: relação da dentição decídua com hábitos e nível socioeconômico. Revista Dental Press de Ortodontia e Ortopedia Facial 1998; 3:35-43.

10. Angle EH. Classification of malocclusion. Dental Cosmos 1899; 41:248-64.

11. Baume LJ. Developmental and diagnostic aspects of the primary dentition. Int Dent J 1959; 9:34966.

12. Maia NG. Prevalência de más oclusões em préescolares da Cidade de Natal na fase de dentição decídua [Dissertação de Mestrado]. Rio Grande do Norte: Faculdade de Odontologia, Universidade Federal do Rio Grande do Norte; 1987.

13. Araujo MCM. Ortodontia para clínicos. São Paulo: Editora Santos; 1986.

14. Moyers RE. Ortodontia. 4a Ed. Rio de Janeiro: Guanabara Koogan; 1991.

15. Organização Mundial da Saúde. Levantamento epidemiológico básico de saúde bucal: manual de instruções. 3a Ed. São Paulo: Editora e Livraria Santos; 1991.

16. Corruccini RS. An epidemiologic transition in dental occlusion in world populations. Am J Orthod 1984; 86:419-26.

17. Brown T. Desenvolvimento e função oclusal nos aborígenes australianos. In: Simões WA, organizador. Ortopedia funcional dos maxilares vista através da reabilitação neuro-oclusal. São Paulo: Editora Santos; 1985. p.1-59.

18. Petrovic AG, Stutzmann JJ. Research methodology and findings applied craniofacial growth studies. In: Graber TM, Rakosi T, Petrovic AG, editors. Dentofacial orthopedics with functional appliances. St. Louis: Mosby; 1997. p. 13-61.

19. Trombelli L, Saletti C, Verna C, Calura G. Prevalenza de carie e malocclusioni in bambini in età scolare della provincia di Ferrara. Mondo Ortod 1991; 16:399-405.

20. Päunio P, Rautava P, Sillanpä ÄM. The finnish family competence study: the effects of living conditions on sucking habits in 3-year-old finnish children and the association between these habits and dental occlusion. Acta Odontol Scand 1993; 51:23-9.

21. Tomita NE. Relação entre determinantes socioeconômicos e hábitos bucais: influência na oclusão de pré-escolares de Bauru-SP - Brasil [Tese de Doutorado]. São Paulo: Faculdade de Odontologia de Bauru, Universidade de São Paulo; 1997.

22. Frazão P. Epidemiologia da oclusão dentária na infância e os sistemas de saúde [Tese de Doutorado]. São Paulo: Faculdade de Saúde Pública, Universidade de São Paulo; 1999.

23. Freeman HP. The meaning of race in science: considerations for cancer research. Cancer 1998; 82:219-25.

24. Graber TM. The finger sucking habit and associated problems. Journal Dental Children 1958; 25:145-51.

25. Bueno APF. Crescimento craniofacial. Rio de Janeiro: Editora do Autor; 1997.

26. Van Norman RA. Digit-sucking: a review of the literature, clinical observations and treatment recommendations. International Journal Orofacial Myology 1997; 23:14-34.

27. Bayardo RE, Mejia JJ, Orozco S, Montoya K. Etiology of oral habits. J Dent Child 1996; 63:350-3.

28. Øgaard B, Larsson E, Lindsten R. The effect of sucking habits, cohort, sex, intercanine arch widths, and breast or bottle feeding on posterior crossbite in Norwegian and Swedish 3-year-old children. Am J Orthod Dentofacial Orthop 1994; 106:161-6.

29. Farsi NMA, Salama FS, Pedo C. Sucking habits in saudi children: prevalence, contributing factors and effects on the primary dentition. Pediatr Dent 1997; 19:28-33.

30. Serra-Negra JMC, Pordeus IA, Rocha Jr. JF. Estudo da associação entre aleitamento, hábitos bucais e mal-oclusões. Rev Odontol Univ São Paulo 1997; 11:79-86.

31. Zuanon ACC. Relação entre hábito bucal e maloclusão na dentadura decídua. JBP J Bras Odontopediatr Odontol Bebê 2000; 3:104-8.

32. De Vis H, De Boever JA, Van Cauwenbergh EP. Epidemiologic survey of functional conditions of the masticatory system in Belgian children aged 3-6 years. Community Dent Oral Epidemiol 1984; 12:203-7.

33. Otuyemi OD, Sote EO, Isiekwe MC, Jones SP. Occlusal relationships and spacing or crowding of 
teeth the dentitions of 3-4-year-old Nigerian children. Int J Paediatr Dent 1997; 7:155-60.

34. Johnson LR. Habits and their relation to malocclusion. J Am Dent Assoc 1943., 30:848-52.

35. Ricketts RM. Respiratory obstruction syndrome. Am J Orthod 1968; 54:495-514.

36. Linder-Aronson S. Respiratory function in relation to facial morphology and the dentition. Br J Orthod 1979; 6:59-71.

37. Cheng MC, Enlow DH, Papsidero M, Broadbent BH, Oyen O. Developmental effects of impaired breathing in the face of the growing child. Angle Orthod 1988; 58:309-20.

38. Emslie RD, Massler M, Zwemer JD. Mouth breathing: etiology and effects (a review). J Am Dent Assoc 1952; 44:506-21.

39. Águila-Ramos FJ, Guzman RA, Rubio EG, Isasi RC. Prevalencia de hábitos bucales deformantes en niños de 3 a 5 años de la ciudad de La Habana. Rev Cubana Estomatol 1980; 17:137-42.

40. Harvold EP, Thomer BS, Vargervik K, Chierici G. Primate experiments on oral respiration. Am J Orthod 1981; 79:359-72.

41. Miller AJ, Vargervick K, Chierici G. Sequential neuromuscular changes in rhesus monkeys during the initial adaptation to oral respiration. Am J Orthod 1982; 81:99-107.

Recebido em 08/Mai/2003

Versão final reapresentada em 01/Out/2003

Aprovado em 10/Out/2003 